\title{
Overcoming the Challenge of Producing Large and Flat Nanocellular Polymers: A Study with PMMA
}

\author{
Judith Martín-de León,* Victoria Bernardo, Paula Cimavilla-Román, Saúl Pérez- \\ Tamarit, and Miguel Ángel Rodríguez-Pérez
}

\begin{abstract}
Although nanocellular polymers are interesting materials with improved properties in comparison with conventional or microcellular polymers, the production of large and flat parts of those materials is still challenging. Herein, gas dissolution foaming process is used to produce large and flat nanocellular polymethylmethacrylate samples. In order to do that, the foaming step is performed in a hot press. The methodology is optimized to produce flat samples with dimensions of $100 \times 100 \times 6 \mathrm{~mm}^{3}$, relative densities in the range $0.25-0.55$ and cell sizes around $250 \mathrm{~nm}$. Additionally, foaming parameters are modified to study their influence on the final cellular structure, and the materials produced in this paper are compared with samples produced by using a most conventional approach in which foaming step is conducted in a thermal bath. Results obtained show that an increment in the foaming temperature leads to a reduction in relative density and an increase of cell nucleation density. Moreover, differences in the final cellular structure for materials produced by both foaming routes are studied, proving that although there exist some differences, the mechanisms governing the nucleation and growing are the same in both processes, leading to the production of homogeneous materials with very similar cellular structures.
\end{abstract}

they also present enhanced mechanical properties, ${ }^{[4]}$ and recently, it has been proved that they can have some new and interesting properties such as transparency. ${ }^{[5]}$

All these properties together make nanocellular polymers the perfect candidates to substitute microcellular materials in sectors like the building sector, the automotive one, in high-performance applications, or in new applications where microcellular materials have no place. However, in order to substitute microcellular materials, it is mandatory to be able to produce nanocellular polymers at a larger-scale.

Gas dissolution foaming process is the most common production method to obtain nanocellular polymers. ${ }^{[6]}$ It consists of three steps, saturation, desorption and the last one, foaming, where samples usually are free foamed in a thermal bath. However, this foaming method does not allow producing large and flat nanocellular polymers, being the dimensions typically smaller than $30 \times 30 \times 4 \mathrm{~mm}^{3} \cdot{ }^{35,7-9]}$ Some previous studies have modified the

\section{Introduction}

Microcellular polymers have been implemented at the industrial level thanks to the successful scaling-up of the production process. Nowadays, large parts of microcellular plastics are produced by means of extrusion or injection molding. ${ }^{[1,2]}$

Although it has been proved that nanocellular polymers perform better than microcellular materials, their production is yet restricted to the laboratory scale, and most of the works on this topic have produced small samples that are not necessarily flat. However, it is already known that nanocellular materials present a smaller thermal conductivity due to Knudsen effect, ${ }^{[3]}$

J. Martín-de León, V. Bernardo, P. Cimavilla-Román, Dr. S. Pérez-

Tamarit, Prof. M. A. Rodríguez-Pérez

Cellular Materials Laboratory (CellMat)

Universidad de Valladolid

47011 Valladolid, Spain

E-mail: jmadeleon@fmc.uva.es

DOI: 10.1002/adem.201900148 last step of the gas dissolution foaming by proposing a constrained foaming process, where the sample is placed between the two heated plates of a hydraulic press. Nadella et al. proposed this method for the production of flat microcellular panels from polymethylmethacrylate (PMMA), polystyrene (PS), and acrylonitrile butadiene styrene (ABS). ${ }^{[10]}$ A similar process was also used by Gedler et al. for the production of graphene-filled polycarbonate composite microcellular foams. ${ }^{[11]}$

However, to the best of our knowledge, this foaming approach has not been previously used for the production of nanocellular polymers, in which due to the higher amount of gas dissolved in the polymer and the critical requirement of not promoting coalescence during growing the process is more critical. This work aims at presenting. for the first time the methodology required to produce larger and flat samples of nanocellular PMMA using a constrained foaming approach in a hot press. Besides, the results obtained in terms of densities and cellular structures are compared with those obtained by using the conventional approach of foaming the samples in a thermal bath. 


\section{Experimental Section}

\subsection{Materials}

The material used in this study was PMMA. The specific polymer, V825T, has been kindly supplied, in the forms of pellets, by ALTUGLAS ${ }^{\circledR}$ International (Colombes, France). The density $(\rho)$ of this polymer is $1.19 \mathrm{~g} \mathrm{~cm}^{-3}$ (measured at $23^{\circ} \mathrm{C}$ and $50 \% \mathrm{HR})$, and its glass transition temperature $\left(T_{\mathrm{g}}\right)$ is $114^{\circ} \mathrm{C}$ (measured by DSC).

Medical grade $\mathrm{CO}_{2}$ (99.9\% purity) was used as blowing agent.

\subsection{Samples Production}

Solid samples of PMMA with dimensions of $60 \times 60 \times 4 \mathrm{~mm}^{3}$ were produced. First of all, the pellets were dried for $4 \mathrm{~h}$ at $80^{\circ} \mathrm{C}$ and then solid precursors of $4 \mathrm{~mm}$ in thickness were produced using a hot plate press from Talleres Remtex. The compression molded process comprises two steps. Pellets were first heated at $250{ }^{\circ} \mathrm{C}$ for $9 \mathrm{~min}$ and then they were pressed under a constant pressure of $54 \mathrm{MPa}$ for $1 \mathrm{~min}$. Finally, they were cooled down at room temperature under the same pressure.

Lastly, the sheets were cut into the desired dimensions for the foaming experiments $\left(55 \times 55 \times 4 \mathrm{~mm}^{3}\right)$.

\subsection{Foaming Tests}

Nanocellular materials were produced by using the gas dissolution foaming process. The set up used for this purpose consists of a high-pressure vessel (model PARR 4681) provided by Parr Instrument Company (Moline, IL, USA) working at a maximum pressure of $40 \mathrm{MPa}$. An accurate pressure pump controller (model SFT-10), provided by Supercritical Fluid Technologies, Inc. (Newark, DE, USA), is available to pressurize the system. Finally, for the foaming step, a hot/cold plate hydraulic press (Talleres Remtex) was used.

The gas dissolution foaming process consists of three steps; saturation, desorption, and foaming. For the first step, samples are introduced in the pressure vessel under a high $\mathrm{CO}_{2}$ pressure atmosphere. This process is extended in time until the sample is fully saturated. Then the pressure is fast released. After a desorption time (time between depressurization and foaming), the sample is foamed in a hot press.

To prevent the expansion of the sample during the desorption time, samples were immediately immersed in ice after releasing the pressure.

The scheme shown in Figure 1 has been followed to foam the samples. The saturated sample is introduced between two steel plates, two aluminum foil sheets and a steel mold of $4 \mathrm{~mm}$ in thickness.

The two steel plates had been previously heated to the desired foaming temperature, while the aluminum foil sheets stay at room temperature in order to prevent any foaming until the sample is under pressure. The steel mold works as a guard to avoid compressing the samples below their initial thickness of $4 \mathrm{~mm}$. Afterward all the layers are introduced between the hot plates of the press, preheated at the foaming temperature, and an

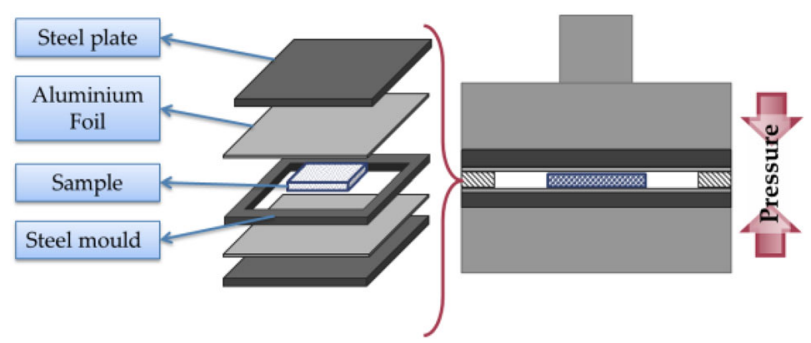

Figure 1. Scheme of the foaming step in a hot press.

initial pressure of $5.4 \mathrm{kPa}$ is applied. As it is discussed later in section 3, the level of this pressure is an important parameter to consider. After the foaming time, the pressure is fast released, and the sample is stabilized.

In this study, the following parameters for the different steps have been chosen. A pressure $\left(p_{\text {sat }}\right)$ of $31 \mathrm{MPa}$ and a temperature $\left(T_{\text {sat }}\right)$ of $25^{\circ} \mathrm{C}$ were selected for the saturation step. Saturation time was selected to be $24 \mathrm{~h}$ for all the experiments. ${ }^{[7]}$ The pressure release was carried out with an electrovalve with $K v=1.11 \mathrm{~min}^{-1}$ at a pressure drop rate of $100 \mathrm{MPas}^{-1}$. Desorption time was 3 min for all the experiments. Finally, foaming conditions were modified; foaming temperatures from 40 to $110^{\circ} \mathrm{C}$ have been tested and foaming times of $0.5 \mathrm{~min}$ and $1 \mathrm{~min}$ were used for each temperature.

Results were compared with those obtained for a set of foamed samples produced using the same saturation conditions, pressure release, and desorption time but foaming using a thermal bath at temperatures from 40 to $110^{\circ} \mathrm{C}$ with foaming times of 1 and 2 min. ${ }^{[12]}$

\subsection{Characterization Techniques}

\subsubsection{Density}

Relative density $\left(\rho_{\mathrm{r}}\right)$, defined as the ratio between the density of the foamed samples $\left(\rho_{\mathrm{f}}\right)$ and the density of the solid samples $\left(\rho_{\mathrm{s}}\right)$, has been determined for all the samples. $\rho_{\mathrm{s}}$ has been measured using a gas pycnometer (Mod. AccuPyc II 1340, Micromeritics, Norcross, GA, USA). On the other hand, previously to determine $\rho_{\mathrm{f}}$, samples were polished in order to remove the solid outer skin ( $0.5 \mathrm{~mm}$ of each side of the samples were removed). Then $\rho_{\mathrm{f}}$ was measured using a density determination kit for an AT261 Mettler-Toledo balance using the water displacement method, based on Archimedes' principle.

\subsubsection{Solubility}

Solubility, defined as the amount of gas uptake, was calculated as the percentage of weight increment of the sample due to the gas sorption. The desorption curve after saturation (mass lost vs. time plot) was registered with a Mettler-Toledo balance and was used to extrapolate to zero time the mass of the samples after saturation. This value is considered as the mass of the sample when it is fully saturated, that is, the solubility of the material. ${ }^{[13]}$ With the previously mentioned saturation conditions $\left(\left(p_{\text {sat }}\right)\right.$ of 
$31 \mathrm{MPa}$ and a temperature $\left(T_{\text {sat }}\right)$ of $\left.25^{\circ} \mathrm{C}\right)$, the reached solubility was $31 \pm 0.3 \%$.

\subsubsection{Scanning Electron Microscopy}

The cellular structure was visualized with an ESEM Scanning Electron Microscope (QUANTA 200 FEG, Hillsboro, OR, USA). Before the visualization, samples were fractured in liquid nitrogen to preserve the original cellular structure. Then they were coated with gold by using a sputter coater (model SDC 005, Balzers Union, Balzers, Liechtenstein).

Cell size in $3 \mathrm{D}(\phi)$, the standard deviation of the cell size distribution divided by the cell size $(\mathrm{SD} / \phi)$, the cell nucleation density $\left(N_{0}\right)$ (calculated by using Kumar's method ${ }^{[6]}$ ) and the anisotropy ratio (AR) were measured to characterize the cellular structure. A software based on Image//FIJI ${ }^{[14]}$ was used for this purpose. Moreover, the mean cell wall thickness $(\xi)$ and the fraction of mass in the struts (fs) were also measured to fully characterize the materials. Cell wall thickness was measured with ImageJ/FIJI directly from the micrographs, while fs was determined following the method described in a previous publication. ${ }^{[12]}$

\subsubsection{Open Cell Content}

The number of open cells in percentage (OC\%) was measured using a gas pycnometer (Mod. AccuPyc II 1340, Micromeritics), according to ASTM D6226-10. Equation (1) was used to calculate it, where $V$ is the geometric volume of the sample, $V_{p}$ is the volume measured by the pycnometer, and $V_{s}$ takes into account the exposed cells at the surface of the sample

$O_{\mathrm{v}}(\%)=\frac{V-V_{\mathrm{p}}-V_{\mathrm{s}}}{V\left(1-\rho_{\mathrm{r}}\right)}$

The external volume was determined as $\underline{V}=m / \rho$ where $m$ is the mass measured with an AT261 Mettler-Toledo balance and $\rho$ is the density determined by the water-displacement method. $V_{\mathrm{p}}$ was determined by using the volume given by the pycnometer at different pressures (from 0.2 $\mathrm{MPa}$ to $1.3 \mathrm{MPa}$ ). From a certain pressure, this value becomes constant. $V_{p}$ was taken as the mean value of these constant values. $V_{s}$ can be neglected for nanocellular materials because this volume is proportional to the cell size.

\subsubsection{Tomography Analysis}

The density distribution in the samples was measured by using X-ray tomography. A homemade set-up with a maximum resolution of 2.5 microns was used to acquire the X-ray tomographies of the samples. ${ }^{[15,16]}$

This method allows detecting in a non-destructive way the presence of internal defects. In addition, it is also possible, to calculate a density profile. This technique allows determining the 3D distribution of attenuation coefficients $(\mu)$ that depends mainly on the density of the materials and the constituent elements. As both gas and polymer are composed mainly by carbon, nitrogen, hydrogen, and oxygen, it is possible to conclude that in our case the attenuation coefficient only depends on materials density without losing generality. As the spatial resolution is not enough to resolve the cellular structure of the analyzed materials, the determined attenuation coefficient in every point of the space (voxel) is a linear combination of the attenuation coefficients of solid $\left(\mu_{s}\right)$ and gas $\left(\mu_{\mathrm{g}}\right)$ constituents in that volume (Equation (2)).

$\mu=X_{\mathrm{s}} \mu_{\mathrm{s}}+\left(1-X_{\mathrm{g}}\right) \mu_{\mathrm{g}}$

where $X_{\mathrm{s}}$ is the volumetric fraction of solid in the considered voxel.

The next step consists of determining the attenuation coefficients of both solid and gas. To this end, a two points calibration process has been implemented (Figure 2).

The first point $\left(\mu_{1}\right)$ is the result of calculating the average attenuation coefficient of the sample considering the full scanned volume. After that, we obtain a first relationship between $\mu_{1}, \mu_{s}$, and $\mu_{g}$ by means of the relative density of the scanned material $\left(\rho_{r}\right)$ (Equation (3)).

$\mu_{1}=\rho_{\mathrm{r}} \mu_{s}+\left(1-\rho_{r}\right) \mu_{\mathrm{g}}$

The second point $\left(\mu_{2}\right)$ is selected out of the sample, consequently is the attenuation coefficient of air. As stated above, since the attenuation coefficient mainly depends on the constituent elements, we can consider that this attenuation coefficient is similar to $\mu_{\mathrm{g}}$ (Equation (4)).

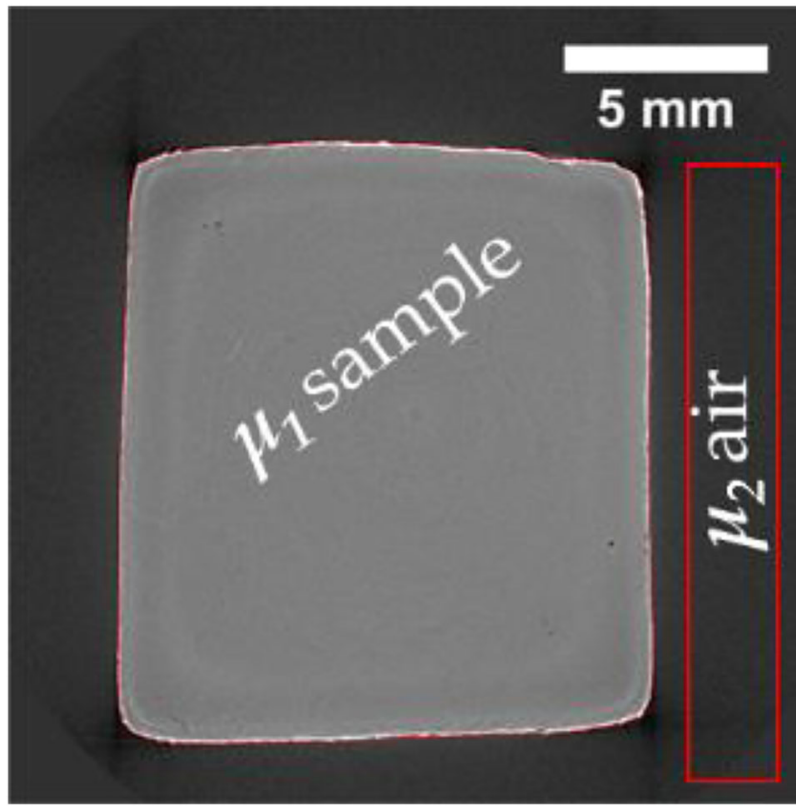

Figure 2. Reconstructed slice of one of the samples showing the two employed zones for the attenuation coefficient calibration. 
$\mu_{2} \approx \mu_{\mathrm{g}} \mu_{1}=\rho_{r} \mu_{s}+\left(1-\rho_{r}\right) \mu_{\mathrm{g}}$

With these two last equations (Equations (2) and (3)), it is possible to finally determine the attenuation coefficient of the solid $\left(\mu_{s}\right)$ (Equation (5)).

$\mu_{s}=\frac{\mu_{1}-\left(1-\rho_{r}\right) \mu_{2}}{\rho_{r}}$

Including now the attenuation coefficients of both solid and gas, the relative density in any point of the sample $\left(\rho_{r}(\vec{r})\right)$ can be calculated (Equation (6)) knowing directly the corresponding attenuation coefficient $(\mu(\vec{r}))$ in that point and those from pure solid and gas calculated using the two selected zones and the Equations (3) and (4).

$\rho_{r}(\vec{r})=\frac{\mu(\vec{r})-\mu_{g}}{\mu_{s}-\mu_{g}}$

\section{Results}

\subsection{External Appearance and Internal Homogeneity of the Samples}

Besides their larger dimensions, all samples produced by means of foaming in a hot press present a flat and homogeneous shape independently of the foaming temperature, being the surface of the sample free of defects. In the thermal bath as the foaming temperature is increased, it is more difficult to maintain the flat shape due to the higher expansion ratio. An example of this is shown in Figure 3b, where two samples foamed at $100^{\circ} \mathrm{C}$ for $1 \mathrm{~min}$ following the two-different foaming routes are shown. For this foaming temperature, the cellular materials have expanded around 4 times with respect the solid precursor (Figure 3a). As it can be observed, while the material produced in the hot press preserve the flat shape, samples produced using the thermal bath are entirely bent and present a significant number of superficial holes.

In order to explore the internal homogeneity of the nanocellular materials, tomography and SEM experiments were performed.

Figure $3 \mathrm{c}$ shows the $3 \mathrm{D}$ density distribution for the samples foamed at $60^{\circ} \mathrm{C}$ for $1 \mathrm{~min}$, as well as a graph showing the relative density of the sample as a function of the thickness. Samples foamed at $60^{\circ} \mathrm{C}$ during $1 \mathrm{~min}$ were chosen for tomography experiments because as it is shown in Figure $3 \mathrm{~b}$ thermal bath samples foamed at $100^{\circ} \mathrm{C}$ presented defects that made more difficult to obtain high-quality tomographic images. For these experiments, the outer surface of the sample was not removed in order to evaluate the modification of the density near the outer surfaces. The upper and the lower part of the hot press foamed samples correspond to those parts in contact with the aluminum foil.

By analyzing the tomography results the expected behavior for the gas dissolution foaming process can be appreciated. ${ }^{[17,18]}$ The central part of the sample is homogeneous and similar for both cellular materials having the lowest density (purplish tones), then when approaching the outer surfaces, the relative density increases up to 1 (yellow tone) indicating the densest zone corresponding to the solid skin.

Nevertheless, it is near the surface where some differences can be appreciated between the two types of samples. The sample foamed in the thermal bath presents a smooth transition from the inner part to the outer skin. However, the sample foamed in the hot press present a more abrupt transition. For this last material, density along the thickness remains constant even near the surface, and therefore near to the solid skin.

To understand this result, it is necessary to analyze the SEM images (Figure 3d). Micrographs with a lower magnification show the samples near the surface. Differences are clear, the sample produced in the hot press shows a transition containing microcells between the solid skin and the nanocellular core. In the sample foamed in the thermal bath, this microcellular transition is almost non-existent, and the number of nanocells gradually decreases up to the solid skin, being this the cause of the soft change in the relative density. However, in the hot press, the microcellular transition makes it possible to maintain the relative density almost constant until the appearance of the solid skin.

Nevertheless, those differences are only shown in the edges of the sample. The central part of the material shows a homogeneous cellular structure (observed in the high magnification SEM images), and therefore an uniform density.

As shown in Figure 3d hot press materials present cell sizes in the nanometric range, very similar to those shown in nanocellular materials foamed in a thermal bath. ${ }^{[12]}$

The explanation of all of this can be found in the thermal transference of both foaming methods. A scheme is shown in Figure 4. In the thermal bath, the temperature of the water is faster transmitted to the sample due to the higher thermal conductivity of this fluid in comparison with air. It means that at a time $t_{1}$ the sample is uniformly hot at a temperature $T_{\mathrm{b}}$ smaller than the final temperature $\left(T_{f}\right)$. The sample homogeneously increases it temperature until reaches $T_{\mathrm{f}}$ at a time $t_{2}$.

On the other hand, the heating of the sample in the hot press is less homogeneous. At a time $t_{1}$, the external parts of the sample heat rapidly at a temperature near $T_{\mathrm{f}}$, while the center of the sample is cooler, at a temperature $T_{\mathrm{p}}<T_{\mathrm{b}}$. This situation evolves up to a time $t_{2}$ where the sample is homogeneous at a temperature $T_{\mathrm{f}}$.

This leads to the results observed in Figure 3. The edges of the samples foamed in the hot press remain longer at a temperature $T_{\mathrm{f}}$ than those ones of the thermal bath samples, resulting in a coalescence of the cells near the surface that creates the microcellular transition between the solid skin and the center of the sample.

As a summary of this section, it can be concluded that the produced materials present a high homogeneity, with slight differences in the density distribution that only affects the areas near the outer skin. A more detailed comparison between the cellular structures in the central region of the samples is included in the following sections. 


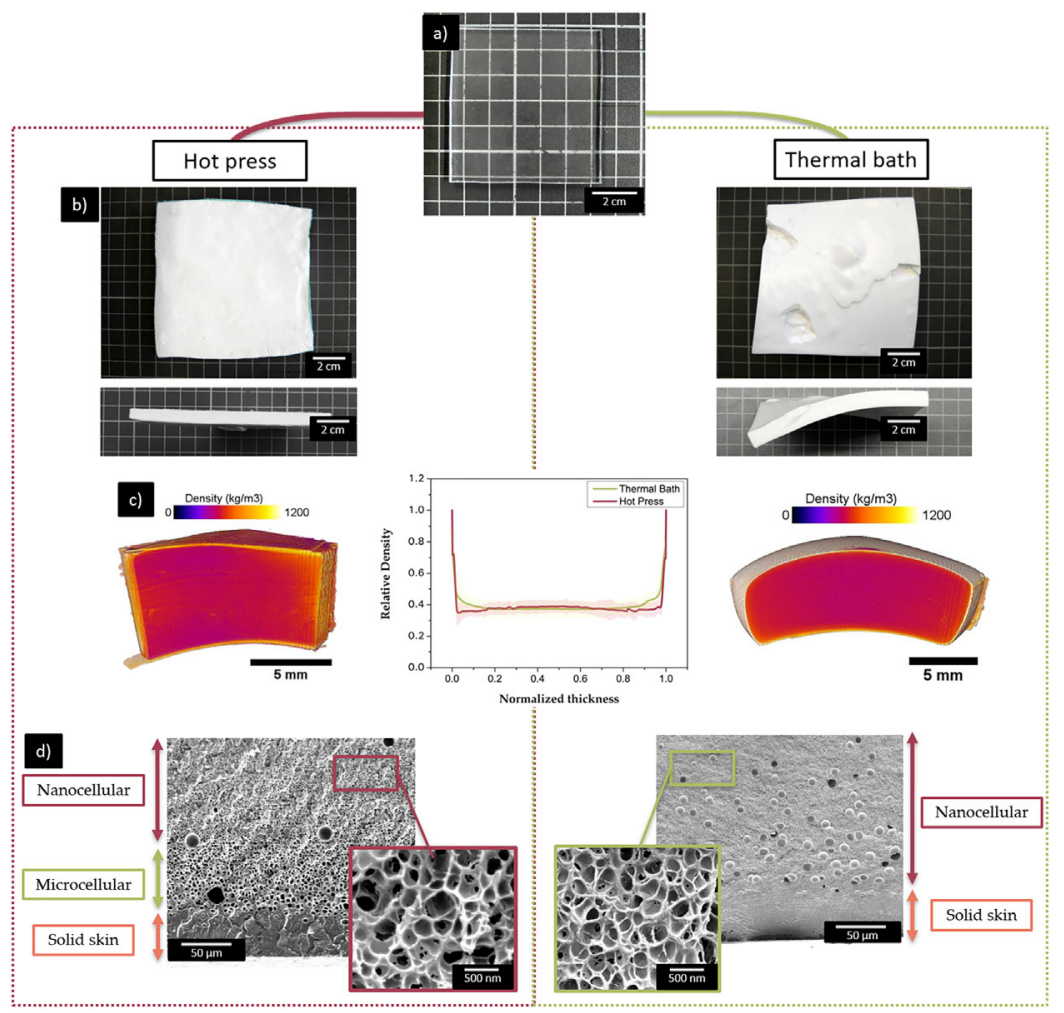

Figure 3. a) Photograph of a solid precursor, b) photograph of the foamed samples at $100^{\circ} \mathrm{C}$ and $1 \mathrm{~min}$. c) Tomography of the foamed samples at $60^{\circ} \mathrm{C}$ and $1 \mathrm{~min}$. d) SEM images of the foamed samples at $60^{\circ} \mathrm{C}$ and $1 \mathrm{~min}$ with two different magnifications.

\subsection{Comparison with Thermal Bath Samples}

\subsubsection{Influence of the Foaming Parameters}

In order to establish the differences between the two different foaming processes, the change in the final cellular structure in the inner part of the samples as a consequence of modifying the foaming parameters has been studied for samples produced by the two methods.
Samples foamed for $1 \mathrm{~min}$, and different foaming temperatures from 40 to $110^{\circ} \mathrm{C}$ were selected for this purpose, and all their characteristics are shown in Table 1.

An increase in the foaming temperature is promoting a decrease in the relative density of all the samples (Figure 5a). In fact, it decreases in a very similar way for the two different foaming methods, starting from around 0.5 when the foaming temperature is $40^{\circ} \mathrm{C}$ and decreasing up to values below 0.3 for temperatures above $80^{\circ} \mathrm{C}$. The differences in density between the two foaming methods are almost negligible in all the temperature range under study, except at $110^{\circ} \mathrm{C}$.

To understand the trends measured for the relative density, attention must be paid to Figure $5 \mathrm{~b}$ in which cell size and cell nucleation density values are summarized. Although changes in relative density are similar for both approaches, some differences in the parameters defining the final cellular structure can be appreciated. Without considering the highest foaming temperature $\left(110^{\circ} \mathrm{C}\right)$, in which cell degeneration mechanisms appear for the samples produced using the thermal bath, it can be asserted that cell size remains constant for both foaming routes when the foaming temperature rises, while cell nucleation density increases. In addition, it is clearly observed that materials produced in the hot press, present values of cell nucleation densities smaller than those foamed in a thermal bath and cell sizes slightly higher.

The cell size distributions are included in Figure $\mathbf{6}$ for samples foamed at 60,80 , and $100^{\circ} \mathrm{C}$. The higher values of cell size for the materials produced in the hot press are a consequence of cell size distributions that are slightly shifted to the right.

Additionally, $\mathrm{SD} / \phi$ and the anisotropy ratio $\mathrm{AR}$ can be observed in Table 1. Both magnitudes present smaller values for the samples foamed in the hot press. It means that although the cell size distribution is shifted to higher values in the hot press

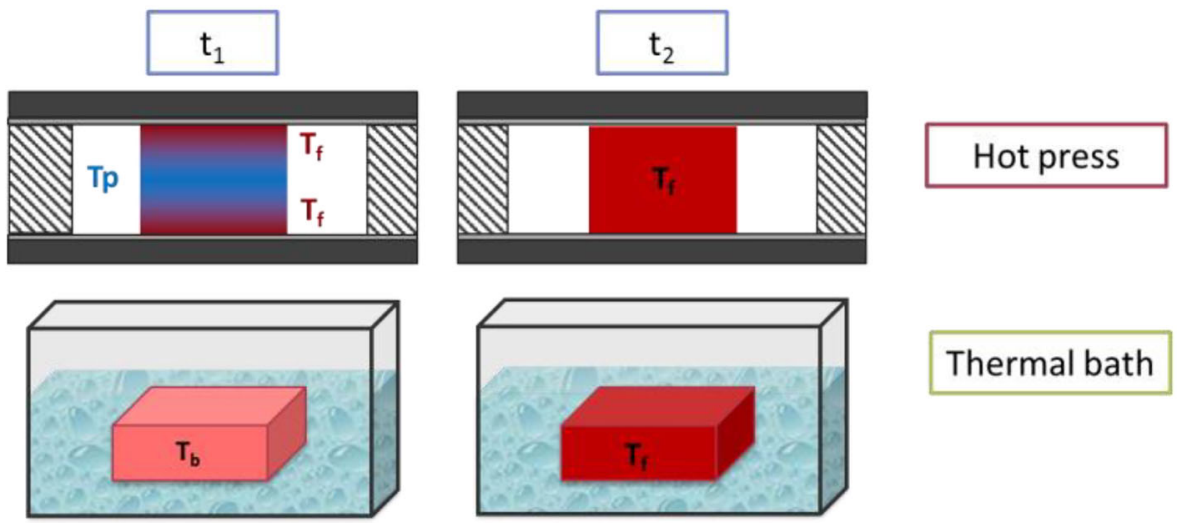

Figure 4. Scheme of the heat transfer for both foaming methods. 
Table 1. Cellular structure characteristics for all the produced samples. The gray saded samples are those ones foamed in the Thermal Bath.

\begin{tabular}{|c|c|c|c|c|c|c|c|c|c|}
\hline Foaming method & Foaming temperature & $\rho_{r}$ & $N_{0}$ [nuclei $\mathrm{cm}^{-3}$ ] & $\varphi[\mathrm{nm}]$ & $\mathrm{SD} / \varphi$ & $A R$ & $f_{\mathrm{s}}$ & $\xi[\mathrm{nm}]$ & OC [\%] \\
\hline Hot press & 40.00 & 0.50 & $1.48 \cdot 10^{14}$ & 258.40 & 0.34 & 1.18 & 0.66 & 31.75 & 5.94 \\
\hline Hot press & 60.00 & 0.40 & $1.42 \cdot 10^{14}$ & 276.69 & 0.39 & 1.12 & 0.59 & 33.45 & 43.47 \\
\hline Hot press & 80.00 & 0.31 & $2.02 \cdot 10^{14}$ & 277.36 & 0.38 & 1.00 & 0.58 & 29.74 & 73.23 \\
\hline Hot press & 100.00 & 0.27 & $2.39 \cdot 10^{14}$ & 257.32 & 0.37 & 1.08 & 0.40 & 28.15 & 72.99 \\
\hline Hot press & 110.00 & 0.31 & $2.84 \cdot 10^{14}$ & 239.74 & 0.38 & 0.99 & 0.42 & 25.65 & 76.97 \\
\hline Thermal bath & 40.00 & 0.47 & $1.78 \cdot 10^{14}$ & 211.83 & 0.45 & 1.24 & 0.60 & 25.52 & 3.48 \\
\hline Thermal bath & 60.00 & 0.39 & $2.38 \cdot 10^{14}$ & 207.41 & 0.42 & 1.21 & 0.54 & 25.93 & 4.53 \\
\hline Thermal bath & 80.00 & 0.27 & $3.56 \cdot 10^{14}$ & 208.12 & 0.45 & 1.21 & 0.35 & 22.89 & 72.93 \\
\hline Thermal bath & 100.00 & 0.27 & $3.44 \mathrm{E}+14$ & 203.86 & 0.41 & 1.18 & -a) & 27.66 & 100.00 \\
\hline Thermal bath & 110.00 & 0.25 & $2.23 E+14$ & 261.08 & 0.43 & 1.19 & -a) & 28.34 & 99.21 \\
\hline
\end{tabular}

a) The missing data for $f_{\mathrm{s}}$ is due to the impossibility of measuring this value in those samples. ${ }^{[12]}$

samples, the distribution is narrower for those samples and moreover, attending to AR they present a smaller anisotropy.

Although classical nucleation theory cannot be used for predicting absolute values of cell size or cell nucleation densities in nanocellular materials, it is possible to explain using this theory the general tendencies observed in our materials. ${ }^{[19]}$ So in order to understand the differences between both foaming routes, the classical homogeneous nucleation theory has been used. ${ }^{[20]}$ For the formation of a nuclei, it is necessary to overcome an energetic barrier given by Equation (7). Also, CNT states that there exists a critical radius below which nuclei do not grow into cells (Equation (8)).

$W=\frac{16 \gamma^{3}}{3 \Delta P^{2}}$

$R_{\mathrm{cr}}=\frac{2 \gamma}{\Delta P}$

Both are functions, among others, of the difference between the pressure inside the bubble and the surrounding system $\Delta P=P_{\text {bub }}-P_{\text {sys }}$.

When there exists stress in the surroundings of the bubbles the expression for the pressure increment is given by
Equation (9). ${ }^{[21,22]}$

$\Delta P=P_{\text {bub }}-\left(P_{\text {sys }}+\Delta P_{\text {local }}\right)$

If the surroundings of the nucleation point experience compressive stress, then $\Delta P_{\text {local }}$ is positive, resulting in an increase of the critical radius as well as the energy barrier for homogeneous nucleation.

This is precisely what is happening in the materials foamed in the hot press; the pressure imposed by the hydraulic press is generating compressive stress that leads to cellular structures with a smaller number of nucleation points than those free foamed in a thermal bath.

For this reason, the control of the parameters of this new foaming process is critical; the applied pressure in the press has to be high enough to maintain the flatness of the sample but not as high to prevent in a significant extend bubble formation. (See supporting information for additional results).

On the other hand, the foaming in a hot press presents additional advantages; as anisotropy ratio indicates it, cellular materials produced by this route are more isotropic than those ones foamed in a thermal bath. As it was aforementioned, solid precursors are produced by compressing the samples in the $Z$ direction. This generates some tensions that result in a small
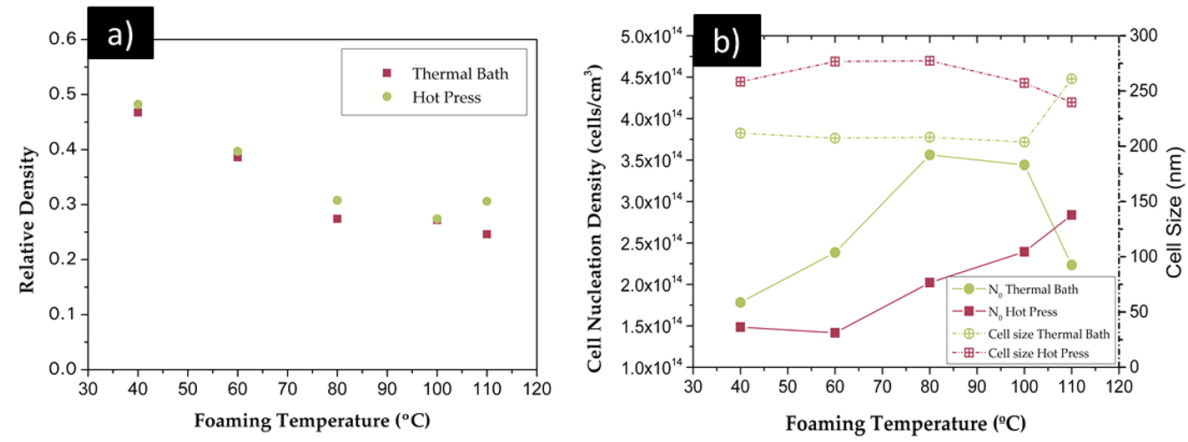

Figure 5. a) Relative density as a function of the foaming temperature. b) Cell size and cell nucleation density as a function of the foaming temperature. Foaming time was $1 \mathrm{~min}$ for all the samples under study. 

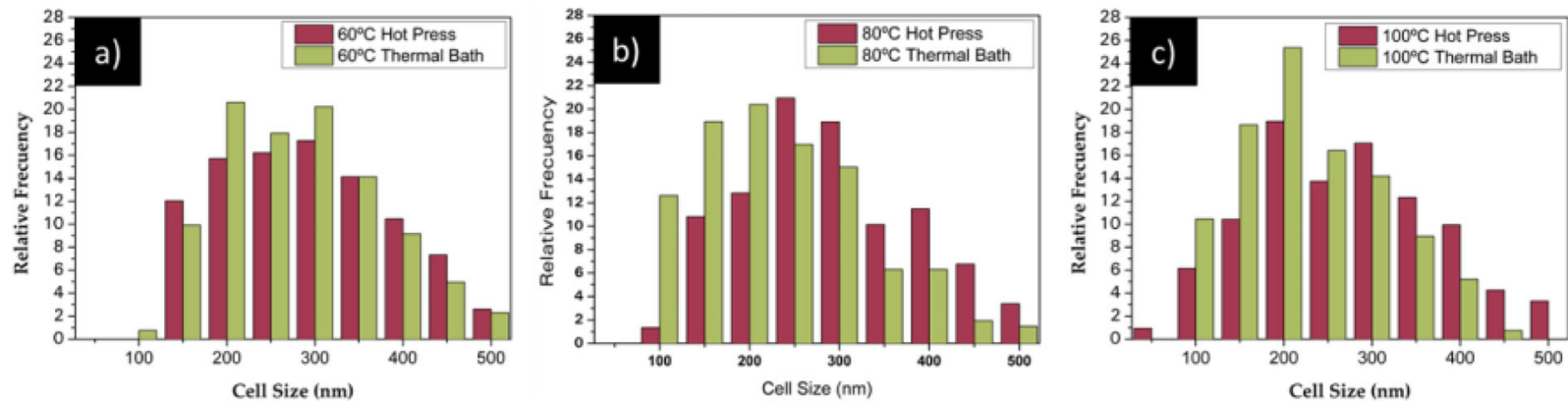

Figure 6. Cell size distributions for samples foamed in a thermal bath and in a hot press at 60 (a), 80 (b), and $100^{\circ} \mathrm{C}$ (c). The foaming time was 1 min for all the samples.

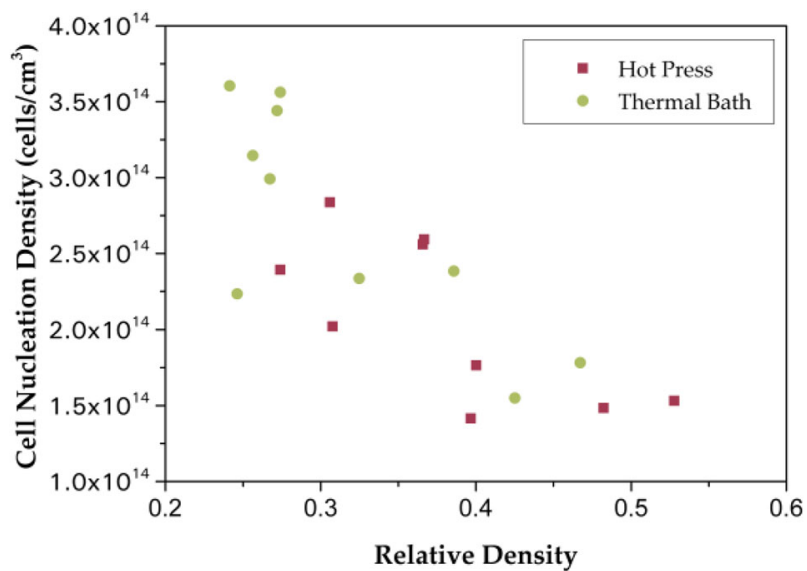

Figure 7. Cell nucleation density as a function of the relative density for samples foamed by the two approaches.

anisotropy when the sample is free foamed in a thermal bath. However, in the hot press, while applying the pressure the sample is able to grow only in the $X Y$ plane, removing this anisotropy effect.

This method is also beneficial when foaming at very high temperatures due to heat transfer. As it was shown in Figure 4 although the surfaces of the sample heated faster, the total heat transfer is slower than in the thermal bath, so when the foaming temperature is as high as $110^{\circ} \mathrm{C}$ the cellular structure degenerates in the thermal bath (Figure $5 \mathrm{~b}$ ) because the matrix easily acquires this temperature and due to the low viscosity coalescence of the cells appears, obtaining a final cellular material with less and bigger cells. In the same conditions, the time for this to happen in the hot press is higher, being possible to still produce nanocellular materials at this temperature and short times of foaming. Therefore, the processing window is wider in the hot press when the foaming temperature is close to the glass transition temperature.

\subsubsection{Foaming Process-Cellular Structure-Density Relationship}

In this section, the changes in the cellular structure are related to the changes in the relative density, in order to investigate in more detail, the two foaming routes.

For the foaming in a thermal bath, it was proved that the mechanism responsible of the reduction of the relative density when foaming temperature increases was the rise in the number of nucleation points while cell size remains constant. This can be observed in Figure 7 together with the data obtained for the hot press foaming that follow a very similar trend. Therefore, it is possible to conclude that the mechanism governing the reduction of the relative density is the same for both foaming routes. Additionally, the hypothesis exposed in the previous section is also visible in this graph, samples foamed in the hot press are not able to reach values of cell nucleation density as high as those obtained in the thermal bath.
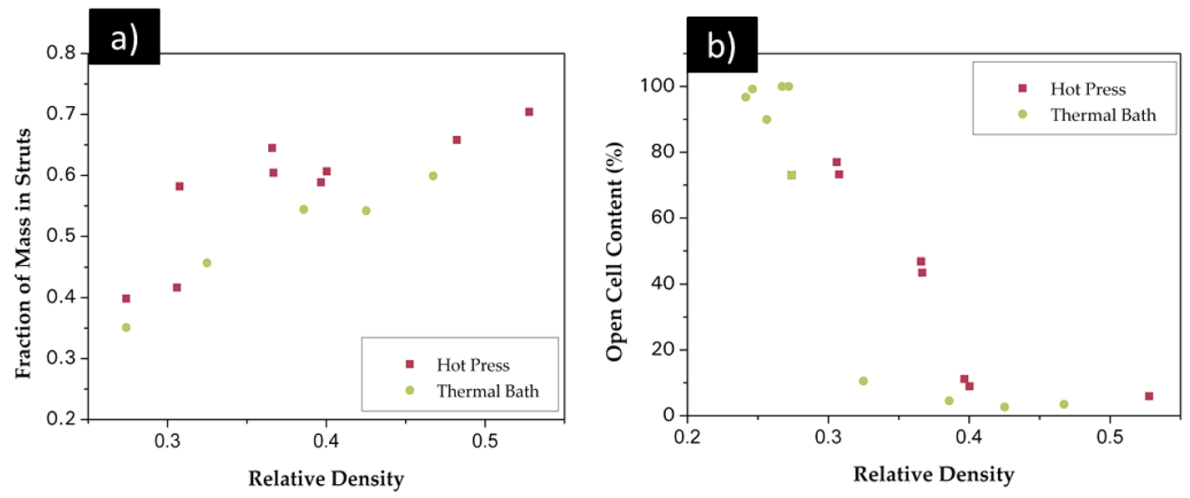

Figure 8. a) Fraction of mass in the struts as a function of the relative density. b) Open cell content as a function of the relative density. 
In addition, as it can be seen in Figure $8 \mathrm{a}$ the reduction of relative density leads to other changes in the cellular structure. As relative density decreases, the fraction of mass in the struts becomes smaller for both foaming processes. On the other hand, open cell content (Figure $8 \mathrm{~b}$ ) increases with the reduction of relative density. For samples presenting the same relative density, slightly higher values of open cell content are found in the hot press samples. However, completely open cell samples have not been produced using the hot press because the minimum relative density achieved is not as small as the one achieved in the thermal bath.

This result indicates that the growth in the $Z$ direction is more abrupt in the case of foaming in the hot press. As it is wellknown, the effective glass transition ( $\left.T_{\text {geff }}\right)$ decreases with the amount of gas uptake. During the foaming, the gas is diffusing out resulting in a gradual increase of the $T_{\text {geff. When foaming in }}$ a thermal bath, this increase is accompanied by a homogeneous growing in all the directions. Nonetheless, in the hot press, the expansion in the $Z$ direction occurs at a higher $T_{\text {geff, }}$ meaning that the polymer is in a more rigid state, this leads to a faster and more abrupt growing in the $Z$ direction, the polymer is deformed at a faster velocity resulting in higher open cell content. On the other hand, this higher $T_{\text {geff }}$ allows the cells to grow less in this direction than in the thermal bath (smaller anisotropy).

\section{Conclusions}

Large and flat nanocellular PMMA parts (sizes of $100 \times 100 \times 6$ $\mathrm{mm}^{3}$ ) have been produced using the gas dissolution foaming process by replacing the common foaming last step of the process consisting on heating the saturated material in a thermal bath by foaming in a hot press.

It has been demonstrated that the nanocellular samples produced in the hot press are very homogeneous both in density and cellular structure.

The cellular structure of the samples foamed in the press is tuneable by changing the foaming temperature. An increase of it leads to smaller relative densities due to an increase in the cell nucleation density. This reduction in the relative density also causes a decrease in the fraction of mass in the struts and an increase of the open cell content. It has been proved that these mechanisms are the same governing the process when the materials are foamed in a thermal bath.

Even though samples produced in the hot press present a slightly inferior number of nucleation sites due to the applied pressure, it is possible to produce nanocellular materials with cell sizes smaller than $250 \mathrm{~nm}$ and relative densities lower than 0.3 .

Therefore, we have proved the concept of producing flat nanocellular polymers with significant sizes. The approach followed could be upscaled to produce even larger samples by using larger solid precursors and a press with a larger surface.

\section{Supporting Information}

Supporting Information is available from the Wiley Online Library or from the author.

\section{Acknowledgements}

Financial assistance from MINECO, FEDER, UE (MAT2015-69234-R) and the Junta de Castile and Leon (VA275P18) are gratefully acknowledged. Financial support from FPU grant FPU14/02050 (V. Bernardo) from the Spanish Ministry of Education and Junta of Castile and Leon grant (J. Martín-de León and P. Cimavilla-Román) are gratefully acknowledged.

\section{Conflict of Interest}

The authors declare no conflict of interest.

\section{Keywords}

flat samples, foaming, gas dissolution foaming, nanocellular polymers, PMMA

Received: February 7, 2019

Revised: April 1, 2019

Published online: April 12, 2019

[1] R. Sriraman, C. Thiagarajan, T. D. Chaudhari, M. Kumar, V. K. Sinha, A. Pattanayak. (2010). Nano-cellular polymer foam and methods for making them. WO Patent ..., 2(12). Retrieved from http:// patentscope.wipo.int/search/en/WO2008087559.

[2] I. Singh, A. Gandhi, M. Biswal, S. Mohanty, S. K. Nayak, Cell. Polym. 2018, 37, 121.

[3] B. Notario, J. Pinto, E. Solorzano, J. A. De Saja, M. Dumon, M. A. Rodriguez-Perez, Polym. (United Kingdom) 2015, 56, 57.

[4] B. Notario, J. Pinto, M. A. Rodriguez-Perez, Prog. Mater. Sci. 2016, 7879, 93.

[5] J. Martín-de León, V. Bernardo, M. Á. Rodríguez-Pérez, Macromol. Mater. Eng. 2017, 3, 1700343.

[6] V. Kumar, N. P. Suh, Polym. Eng. Sci. 1990, 30, 1323.

[7] H. Guo, V. Kumar, Polymer (Guildf). 2015, 57, 157.

[8] V. Kumar, J. Weller, J. Eng. Ind. 1994, 116, 413.

[9] V. Bernardo, J. Martín-De León, M. A. Rodríguez-Pérez, Mater. Lett. 2016, 178, 155.

[10] K. Nadella, V. Kumar, W. Li, Cell. Polym. 2005, 24, 71.

[11] G. Gedler, M. Antunes, J. I. Velasco, J. Supercrit. Fluids 2014, $88,66$.

[12] J. Martín-de León, V. Bernardo, M. Rodríguez-Pérez, Polymers (Basel). 2016, 8, 265.

[13] H. Guo, V. Kumar, Polymer (Guildf). 2015, 56, 46.

[14] J. Pinto, E. Solorzano, M. A. Rodriguez-Perez, J. A. de Saja, J. Cell. Plast. 2013, 49, 555.

[15] E. Solórzano, J. Pinto, S. Pardo, F. Garcia-Moreno, M. A. RodriguezPerez, Polym. Test. 2013, 32, 321.

[16] S. Pérez-Tamarit, E. Solórzano, A. Hilger, I. Manke, M. A. RodríguezPérez, Eur. Polym. J. 2018, 109, 169.

[17] J. Pinto, S. Pardo, E. Solórzano, M. a. Rodríguez-Pérez, M. Dumon, J. a. de Saja, Defect Diffus. Forum 2012, 326-328, 434.

[18] V. Kumar, J. E. Weller, Polym. Eng. Sci. 1994, 34, 169.

[19] S. Costeux, J. Appl. Polym. Sci. 2015, 132, n/a.

[20] L. J. Gibson, M. F. Ashby, Cellular Solids: Structure and Properties, Cambridge: Cambridge University Press, United States 1997.

[21] R. J. Albalak, Z. Tadmor, Y. Talmon, AlChE J. 1990, 36, 1313.

[22] S. N. S. Leung, Ph. D. Thesis 2009, 239. 